\title{
Local overexpression of HB-EGF exacerbates remodeling following myocardial infarction by activating noncardiomyocytes
}

\author{
Hiroaki Ushikoshi, ${ }^{1,2}$, Tomoyuki Takahashi ${ }^{1,4,5}$, Xuehai Chen ${ }^{2}$, Ngin Cin Khai ${ }^{1,2}$, \\ Masayasu Esaki ${ }^{1,2}$, Kazuko Goto ${ }^{1,2}$, Genzou Takemura ${ }^{2}$, Rumi Maruyama ${ }^{2}$, \\ Shinya Minatoguchi ${ }^{2}$, Takako Fujiwara ${ }^{3}$, Satoshi Nagano ${ }^{1,4}$, Kentaro Yuge ${ }^{1}$, \\ Takao Kawai ${ }^{1,2}$, Yoshiteru Murofushi ${ }^{1,4}$, Hisayoshi Fujiwara ${ }^{2}$ \\ and Ken-ichiro Kosai ${ }^{1,4,6}$
}

${ }^{1}$ Department of Gene Therapy and Regenerative Medicine, Gifu University School of Medicine, Gifu, Japan; ${ }^{2}$ Department of Cardiology, Respirology and Nephrology, Regeneration \& Advanced Medical Science, Gifu University Graduate School of Medicine, Gifu, Japan; ${ }^{3}$ Department of Food Science, Kyoto Women's University, Kyoto, Japan; ${ }^{4}$ Division of Gene Therapy and Regenerative Medicine, Cognitive and Molecular Research Institute of Brain Diseases, Kurume University, Kurume, Japan; ${ }^{5}$ Department of Advanced Therapeutics and Regenerative Medicine, Kurume University School of Medicine, Kurume, Japan and ${ }^{6}$ Department of Pediatrics and Child Health, Kurume University School of Medicine, Kurume, Japan

\begin{abstract}
Insulin-like growth factor (IGF), hepatocyte growth factor (HGF), and heparin-binding epidermal growth factorlike growth factor (HB-EGF) are cardiogenic and cardiohypertrophic growth factors. Although the therapeutic effects of IGF and HGF have been well demonstrated in injured hearts, it is uncertain whether natural upregulation of HB-EGF after myocardial infarction (MI) plays a beneficial or pathological role in the process of remodeling. To answer this question, we conducted adenoviral HB-EGF gene transduction in in vitro and in vivo injured heart models, allowing us to highlight and explore the HB-EGF-induced phenotypes. Overexpressed HB-EGF had no cytoprotective or additive death-inducible effect on Fas-induced apoptosis or oxidative stress injury in primary cultured mouse cardiomyocytes, although it significantly induced hypertrophy of cardiomyocytes and proliferation of cardiac fibroblasts. Locally overexpressed HB-EGF in the MI border area in rabbit hearts did not improve cardiac function or exhibit an angiogenic effect, and instead exacerbated remodeling at the subacute and chronic stages post-MI. Namely, it elevated the levels of apoptosis, fibrosis, and the accumulation of myofibroblasts and macrophages in the MI area, in addition to inducing left ventricular hypertrophy. Thus, upregulated HB-EGF plays a pathophysiological role in injured hearts in contrast to the therapeutic roles of IGF and HGF. These results imply that regulation of HB-EGF may be a therapeutic target for treating cardiac hypertrophy and fibrosis.
\end{abstract}

Laboratory Investigation (2005) 85, 862-873. doi:10.1038/labinvest.3700282; Published online 25 April 2005

Keywords: apoptosis; gene transfer; growth factor; myocardial infarction; remodeling

Heparin-binding epidermal growth factor-like growth factor (HB-EGF), a member of the EGFfamily of growth factors, is synthesized as a type I transmembrane protein (proHB-EGF). ${ }^{1}$ Membranebound proHB-EGF is cleaved at its juxtamembrane

Correspondence: Dr K Kosai, MD, PhD, Division of Gene Therapy and Regenerative Medicine, Cognitive and Molecular Research Institute of Brain Diseases, Kurume University, 67 Asahi-machi, Kurume 830-0011, Japan.

E-mail: kosai@med.kurume-u.ac.jp

Received 27 November 2004; revised 9 March 2005; accepted 15 March 2005; published online 25 April 2005 domain by a specific metalloproteinase, resulting in shedding of soluble HB-EGF. ${ }^{2}$ Whereas soluble HBEGF is a potent mitogen for a number of cell types, including vascular smooth muscle cells, fibroblasts, keratinocytes, and hepatocytes, ${ }^{3-5}$ the activity of proHB-EGF may be mitogenic or growth inhibitory depending on cell type. ${ }^{6}$

HB-EGF has been implicated in a number of physiological and pathological processes. HB-EGF may play a role in the development of atherosclerosis resulting from smooth muscle cell hyperplasia, ${ }^{4,7,8}$ pulmonary hypertension, and oncogenic transformation. ${ }^{9,10}$ In contrast, HB-EGF is 
therapeutic for the skin, ${ }^{11,12}$ kidney, ${ }^{13,14}$ liver, and small intestine. ${ }^{3,5,15,16}$ HB-EGF is markedly upregulated during the acute phase of injury and plays an essential role in epithelial cell repair, proliferation and regeneration in these organs. ${ }^{5,11,13,15,17}$ Further direct evidence of therapeutic benefit was provided by studies of administration of recombinant HB-EGF in animal ischemic disease models. ${ }^{16}$ Thus, HB-EGF plays a number of physiological roles, and its effects are diverse and even opposing in nature depending on the tissues examined.

It has been observed that HB-EGF-null mice develop severe heart failure associated with dilated ventricular chambers, diminished cardiac function, and grossly enlarged cardiac valves, ${ }^{18,19}$ indicating that HB-EGF is an essential cardiogenic factor. HBEGF is found in the adult heart under normal physiological conditions, ${ }^{20}$ and the HB-EGF and/or EGF receptor (EGFR) families are further upregulated under pathological conditions such as cardiac hypertrophy ${ }^{21}$ or myocardial infarction (MI). ${ }^{22,23}$ Together with the recent finding that shedding of proHB-EGF results in cardiac hypertrophy, ${ }^{24}$ it has recently been suggested that HB-EGF-induced cardiomyocyte hypertrophy plays a central role in hypertensive heart disease. ${ }^{24,25}$ However, several previous studies demonstrated that overexpression of hepatocyte growth factor (HGF) and insulin-like growth factor (IGF), which are also cardiogenic growth factors, significantly induced cardiac hypertrophy but had potent therapeutic rather than pathologic effects in injured hearts, including those damaged by MI. ${ }^{26-29}$ This led us to question whether HB-EGF might also possess therapeutic activity in the injured heart. Intriguingly, were HB-EGF to prove pathogenic, it could be the result of a secondary biological effect of this molecule separate from its promotion of hypertrophy. Thus, we endeavored to settle the questions raised by these conflicting reports in the most direct way possible, through targeted overexpression of HB-EGF in heart lesions.

One obvious approach by which to overexpress a target gene and explore the resulting effects would be to use transgenic mouse (TgM) technology, currently one of the most powerful approaches to elucidate directly the physiological and pathological roles of a gene of interest. In the present study, we opted instead to use an adenoviral gene transduction strategy, which allowed us to answer the same biological question while at the same time enabling a first assessment of the use of HB-EGF in gene therapy. Additionally, the use of an adenoviral vector allowed for greater spatial and temporal control of HB-EGF expression compared with the TgM approach, as persistent overexpression of the transgene (from the embryonic stage and before the onset of a disorder) may have produced data artefacts. Previous studies demonstrated that the expression of HB-EGF and EGFR family mRNAs was significantly increased around MI lesions. ${ }^{22,23}$ In this context, adenoviral HB-EGF gene transduction around the MI area following onset of MI may serve to highlight the effect of HB-EGF on both cardiomycytes and noncardiomyocytes following MI, offering a means to elucidate the role of this intriguing molecule in the development of heart disease.

\section{Materials and methods}

\section{Recombinant Adenoviral Vectors}

Replication-defective recombinant adenoviral vectors (Ads), Ad.HB-EGF and Ad.LacZ, which express HB-EGF or LacZ gene under the transcriptional control of a Rous sarcoma virus long-terminal repeat, were constructed as described previously. ${ }^{27,30,31}$ All Ads were amplified in 293 cells, purified twice on $\mathrm{CsCl}$ gradients, and desalted..$^{27,30,31}$

\section{Injury Models in Primary Cultured Cardiomyocytes and Cardiac Fibroblasts}

Cardiomyocytes and cardiac fibroblasts were isolated from 1-day-old neonatal Balb/c mice as previously reported..$^{32}$ The cardiomyoctes were incubated in Dulbecco's modified Eagle's medium (D-MEM, Sigma Chemical Co., St Louis, MO, USA) containing 5\% fetal bovine serum (Sigma Chemical Co.) at $37^{\circ} \mathrm{C}$ for $24 \mathrm{~h}$, and subsequently infected with Ads at various multiplicities of infection (MOI), followed by incubation in serum-free D-MEM for $48 \mathrm{~h}$. In injury models of apoptosis and oxidative stress, cells were incubated with either $1 \mu \mathrm{g} / \mathrm{ml}$ agonistic anti-Fas antibody ${ }^{33}$ (Jo2, Beckton-Dickinsion Biosciences, San Jose, CA, USA) with $0.05 \mu \mathrm{g} /$ $\mathrm{ml}$ actinomycin D (Sigma Chemical Co.) for $24 \mathrm{~h}$, or with $100 \mu \mathrm{M} \mathrm{H}_{2} \mathrm{O}_{2}$ (Wako Pure Chemical Industry, Osaka, Japan) for $1 \mathrm{~h}$ as previously described. ${ }^{34,35}$ Cell viability was determined by WST-8 assay (Dojindo, Kumamoto, Japan) in accordance with the manufacturer's protocol $24 \mathrm{~h}$ after the induction of cell death.

For proliferation assays, cardiac fibroblasts were incubated in D-MEM supplemented with $5 \%$ fetal bovine serum, and were used following three or four passages. The purity of these cultures was $>95 \%$ cardiac fibroblasts as confirmed by vimentin-positive, desmin-negative and $\alpha$-smooth muscle actinnegative stainings as previously described. ${ }^{36}$ WST-8 assay was performed at 24,48 and $72 \mathrm{~h}$ after infection with Ads or addition of recombinant human HB-EGF (R\&D Systems Inc., Minneapolis, MN, USA).

\section{Immunocytochemistry and Analysis of Primary Cultured Cardiomyocytes}

At $24 \mathrm{~h}$ following adenoviral infection at MOI 30 , primary cultured cardiomyocytes were fixed in $4 \%$ paraformaldehyde, permeabilized with $0.05 \%$ 
Triton-X and stained with primary goat anti-human HB-EGF antibody (R\&D Systems Inc.), secondary donkey anti-goat IgG Alexa 488 antibody (Molecular Probes, Inc., Eugene, OR, USA), rhodamine phalloidin (Molecular Probes, Inc.) and Hoechst 33342 (Molecular Probes, Inc.). Digital images captured using a laser-confocal microscope system (LSM510, Carl Zeiss, Oberkochen, Germany) were employed for morphometric and quantitative analyses using Adobe Photoshop 7.0 software (Adobe Systems Inc., San Jose, CA, USA).

\section{Animal Studies}

Male Japanese white rabbits weighing $2-2.5 \mathrm{~kg}$ underwent a 30-min occlusion of the left coronary artery, followed by reperfusion, in order to generate MI as previously described. ${ }^{37}$ Ad.HB-EGF or control Ad.LacZ $\left(1 \times 10^{11}\right.$ viral particles) (each group, $n=16$ ) was directly injected into the border area between the risk and the intact areas at the time of reperfusion. Echocardiograms were recorded just before and 2 or 4 weeks after generation of MI. The rabbits were killed either 2 or 4 weeks later (each, $n=8$ ) and the hearts were collected, weighed, and then processed to obtain histological sections. In the sham control group $(n=4)$, the chests of the rabbits were opened and closed under anesthesia without occlusion of the coronary artery or adenoviral injection, and echocardiograms and histological analyses were performed 2 or 4 weeks later. All animal studies were performed in accordance with the guidelines of the National Institute of Health as dictated by the Animal Care Facility at the Gifu University School of Medicine.

\section{Adenoviral Gene Transduction Efficiencies and X-Gal Staining}

The efficiency of in vitro and in vivo adenoviral gene transduction was analyzed by Ad.LacZ infection and X-gal staining, as previously described. ${ }^{27,30,31}$

\section{Pathological Examination in Animal Experiments}

The estimation of the risk and MI areas has been described previously. ${ }^{37}$ Briefly, the coronary branch in the excised heart was reoccluded and 4\% Evans blue dye (Sigma Chemical Co.) was injected via the aorta to determine the risk area. The LV was sectioned into seven slices parallel to the atrioventricular ring. Each slice was incubated in 1\% solution of triphenyl tetrazolium chloride (TTC) to visualize the infarct area.

For histological analysis, the heart was fixed in $10 \%$ formalin and embedded in paraffin, and $4-\mu \mathrm{m}$ sections were stained with hematoxylin and eosin (H-E) or Masson's trichrome for regular or fibrotic estimation, respectively. The sizes of individual cardiomyocytes were measured using the LUZEX F system (Nireco, Kyoto, Japan). Apoptotic cells were detected under light microscopy by terminal deoxynucleotidyl transferase-mediated deoxyuridine triphosphate biotin nick end labeling (TUNEL) assay (ApopTag kit, Intergen Co., Purchase, NY, USA) in accordance with the manufacturer's protocol. The immunohistochemical staining for proliferating cells, $\alpha$-smooth muscle actin (SMA-positive cells), rabbit macrophages and vascular endothelial cells were carried out with anti-Ki-67 (MIB-1, Dako), anti-SMA (1A4, Dako), anti-RAM11 (Dako) and anti-CD31 (JC/70A, Dako) antibody, respectively, as described previously. ${ }^{37}$ For fluorescent immunohistochemistry and TUNEL assay, 6- $\mu \mathrm{m}$ frozen sections were fixed in $4 \%$ paraformaldehyde and stained using a fluorescein-FragEL DNA fragmentation detection kit (Oncogene Research Products, San Diego, CA, USA) together with individual antibodies, according to the manufacturer's instructions.

\section{Statistical Analysis}

Data are represented as means \pm standard error of the mean. Statistical significance was determined using Student's $t$-test. One-way ANOVA was used in multiple comparisons. $P<0.05$ was considered to be statistically significant. All statistical analysis was performed with StatView software (SAS Institute Inc., Cary, NC, USA).

\section{Results}

\section{Adenoviral Gene Transduction Efficiency In Vitro}

The adenoviral constructs demonstrated high infectivity in primary cultured mouse cardiomyocytes; infection of cardiomyocytes with Ad.LacZ at MOIs of 10 and 30 resulted in approximately $80 \%$ and over $90 \%$ successful gene transduction, respectively, without morphological changes, cell damage or death (Figure 1). Cardiac fibroblasts also demonstrated high infectivity, although the accurate quantification was difficult.

\section{Effects of Adenoviral HB-EGF Gene Transduction on Cardiomyocytes and Cardiac Fibroblasts In Vitro}

To explore the direct effects of HB-EGF on cardiomyocytes, we examined cell viability following Ad.HB-EGF infection in two representative injury models of primary cultured cardiomyocytes, Fasinduced apoptosis ${ }^{34,35}$ and $\mathrm{H}_{2} \mathrm{O}_{2}$ oxidative stress injury $^{34,35}$ (Figure 2a and b). Both types of stimulus at the predetermined doses efficiently induced cell death in approximately $80 \%$ of the cultured cells, and adenoviral HB-EGF gene transduction did not result in significant changes in viability in either of these models at any MOI (Figure 2a and b). However, 

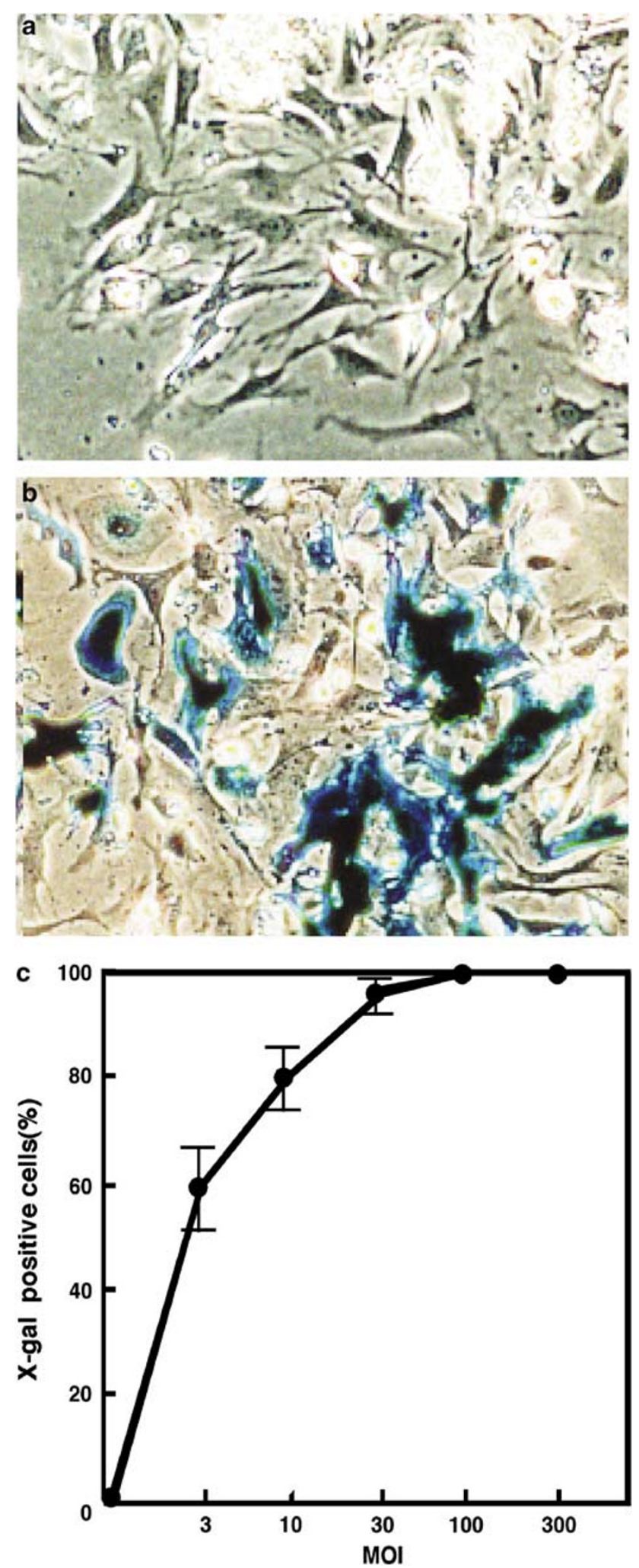

Figure 1 Efficiency of adenoviral gene transduction in primary cultured mouse cardiomyocytes. X-gal staining after Ad.LacZ infection at (a) MOI 0 (ie, no infection as a negative control) and (b) MOI 10. (c) Graph showing adenoviral gene transduction efficiency at various MOIs.
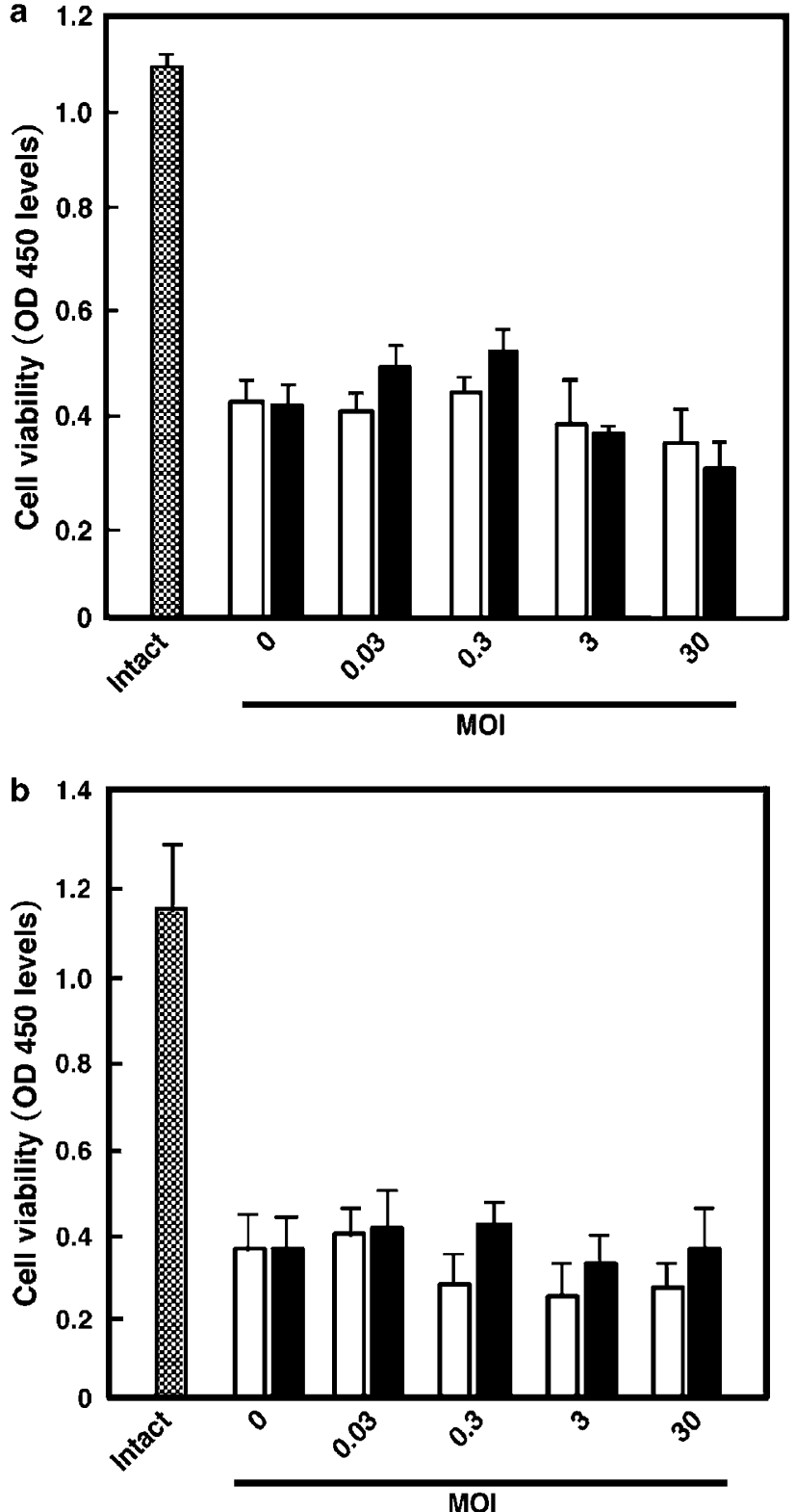

Figure 2 Cell viability after adenoviral HB-EGF gene transduction in two injury models of primary cultured cardiomyocytes. Primary cultured cardiomyocytes were infected with Ad.LacZ (white bar) or Ad.HB-EGF (black bar), and were then exposed to $1 \mu \mathrm{g} / \mathrm{ml}$ anti-Fas antibody and $0.05 \mu \mathrm{g} / \mathrm{ml}$ actinomycin D for $24 \mathrm{~h}$ (a) or $100 \mu \mathrm{M} \mathrm{H}_{2} \mathrm{O}_{2}$ for $1 \mathrm{~h}$ (b); cell viability was evaluated by WST8 assay. 'Intact' indicates the control (untreated cells).

cardiomyocytes became significantly enlarged, and their F-actin-containing myofibrils were drastically condensed, enlarged and increased in number following adenoviral HB-EGF gene transduction (Figures 3 and 4).

Next, we explored whether HB-EGF exhibited an inhibitory or stimulatory effect on the growth of cardiac fibroblasts. Both the addition of recombinant HB-EGF and adenoviral HB-EGF gene transduction significantly accelerated the growth of cardiac fibroblasts. (Figure 5). Thus, HB-EGF gene 
866
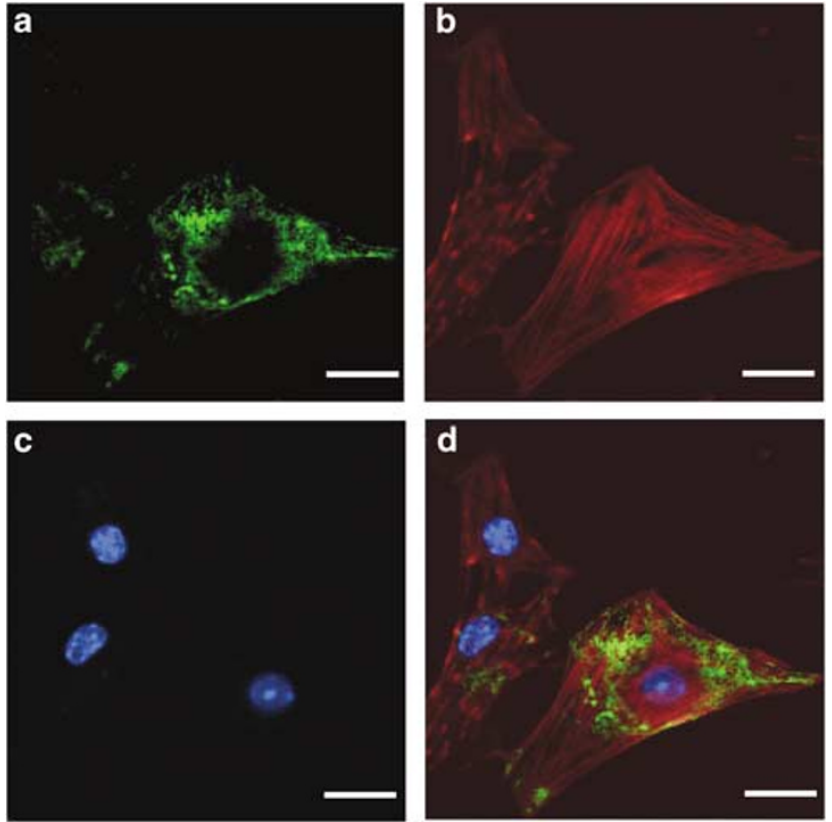

Figure 3 Microscopic images of HB-EGF gene-transduced cardiomyocytes. Confocal microscopic analysis demonstrated apparent hypertrophic changes in individual cardiomyocytes overexpressing HB-EGF after Ad.HB-EGF infection at MOI 30. (a) Staining with goat anti-human HB-EGF antibody and Alexa 488-labeled donkey anti-goat secondary antibody, (b) staining using rhodamine phalloidin-labeled F-actin, (c) staining using Hoechst 33342 (nuclei), and (d) merged image. Scale bar $=20 \mu \mathrm{m}$.
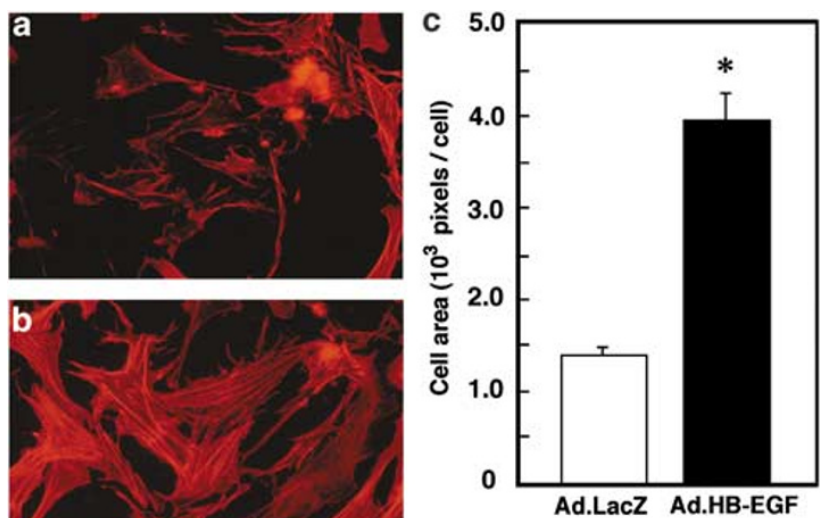

Figure 4 Morphometric and quantitative analysis of HB-EGF gene-transduced cardiomyocytes after Ad.LacZ (a) or Ad.HB-EGF (b) infection at MOI 30. Hypertrophy as shown by condensation of rhodamine phalloidin-labeled F-actin and enlargement of cell area. Original magnification, $\times 100$. (c) Graphic depiction of cell area determined for 500 cardiomyocytes infected with Ad.HBEGF or Ad.LacZ. ${ }^{*} P<0.001$.

transduction and overexpression conferred a direct hypertrophic effect on cardiomyocytes and a growth-stimulating effect on cardiac fibroblasts, but did not have additive death-inducible or cytoprotective effects on cardiomyocytes.

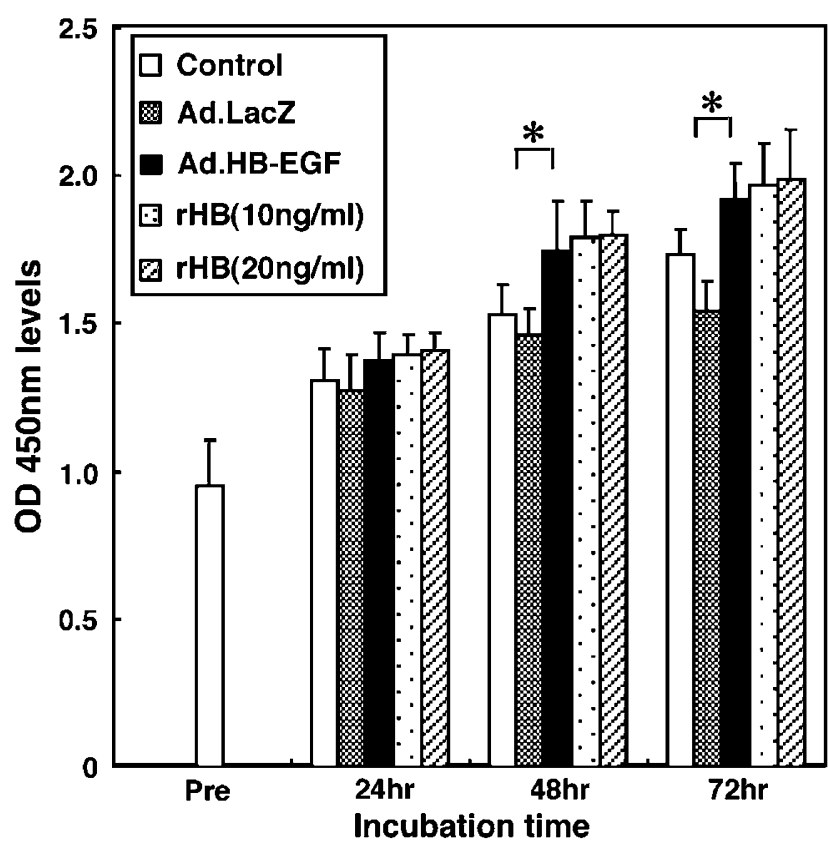

Figure 5 Proliferation of cardiac fibroblasts overexpressing HBEGF. Cardiac fibroblasts (cell density; $1 \times 10^{5} / \mathrm{ml}$ ) were infected with Ad.LacZ at MOI 30 (shaded bar) or Ad.HB-EGF at MOI 30 (black bar), or were exposed to 10 or $20 \mathrm{ng} / \mathrm{ml} \mathrm{rHB-EGF} \mathrm{for} 24,48$, and $72 \mathrm{~h}$; cell proliferation was evaluated by WST-8 assay at OD $450 \mathrm{~nm}$. ${ }^{*} P<0.05$.

\section{Macroscopic Findings after Adenoviral HB-EGF Gene Transduction in the Rabbit MI Model}

Recent studies have demonstrated that HB-EGF and EGFR family mRNAs were significantly upregulated around MI lesions, ${ }^{22,23}$ and it is for this reason that we injected our adenovirus constructs specifically into this region. X-gal staining after Ad.LacZ injection into this area showed that we were able to drive gene transduction predominantly around the MI (Figure 6a). A number of previous studies have demonstrated retention of transgene expression for at least 3 weeks following in vivo adenoviral gene transduction into the heart. ${ }^{38-40}$ We have previously described the pathological process in the rabbit MI model in detail, including granulation consisting of myofibroblasts, macrophages and neovasculation at 2 weeks (the subacute stage) and scar formation at 4 weeks (the chronic stage). ${ }^{37}$ Together, these results suggested that the period of transgene expression resulting from adenoviral gene transduction would be sufficient for the purposes of this study. In this context, we injected Ad.HB-EGF into the MI injury border area, estimated the risk area and the MI area by TTC and Evans blue dye after 2 weeks (as shown in Figure 6b) and ultraechographically analyzed cardiac function 2 and 4 weeks later. There was no difference in risk area between the two groups (Figure 6c). On the other hand, the ratio of MI area to risk area was seemingly reduced to a small degree by Ad.HB-EGF at 2 weeks post-injection due to hypertrophic changes; however, this reduction was 

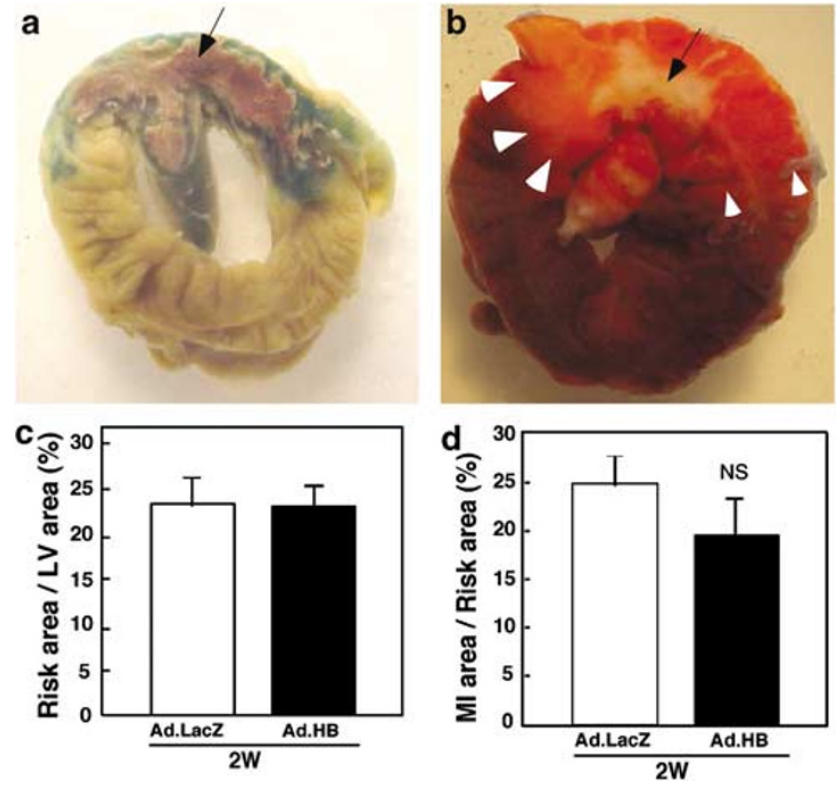

Figure 6 Macroscopic findings after adenoviral HB-EGF gene transduction in a rabbit MI model. (a) X-gal staining after Ad.LacZ injection into the border area. The arrow indicates the infarcted area. (b) Triphenyl tetrazolium chloride (TTC) and Evans blue staining define the risk areas (arrowheads), MI areas (arrow), and the intact (blue) areas. Graphs showing the risk area (c) and the MI area (d). Morphometric analysis of TTC/Evans bluestained macroscopic slides 2 weeks after MI. NS: no significant difference.

not statistically significant (HB-EGF; $19.6 \pm 3.5 \%$ vs LacZ; $24.6 \pm 2.9 \%, P=0.277$ ) (Figure 6d).

\section{Cardiac Function and Histological Changes Following Adenoviral HB-EGF Gene Transduction in the Rabbit MI Model}

Rabbits that received a control Ad.LacZ injection following MI clearly demonstrated a worsening of cardiac parameters such as left ventricular ejection fraction (LVEF) and left ventricular dimension at end-diastole (LVDd), as assessed by ultraechocardiography (UCG) at 2 and 4 weeks post-MI, compared with sham-operated rabbits that underwent neither adenoviral gene transduction nor MI (Figure 7a and b). Ad.HB-EGF injection after MI neither improved nor further worsened cardiac function, as assessed by LVEF or LVDd at 2 or 4 weeks compared with the Ad.LacZ-treated rabbits. On the other hand, anterior wall thickness (AWt) at 2 weeks (HB-EGF, $2.7 \pm 0.3 \mathrm{~mm}$ vs LacZ, $1.9 \pm 0.1 \mathrm{~mm}, \quad P<0.05$, Figure $7 \mathrm{c}$ ) and the ratio of $\mathrm{LV}$ weight to body weight at 2 and 4 weeks were significantly increased by Ad.HB-EGF injection (2 weeks: HB-EGF, 1.59 0.06 vs LacZ, $1.44 \pm 0.03, P<0.05 ; 4$ weeks: HB-EGF, $1.67 \pm 0.09$ vs LacZ, $1.40 \pm 0.05, P<0.05$ ) (Figure $7 \mathrm{~d}$ ); these findings were consistent with macroscopically observed hypertrophic changes (Figure 8). In addition,

fibrosis in and around the MI area, which was induced by the MI itself, was increased by injection with Ad.HB-EGF at 2 and 4 weeks post-MI (2 weeks: HB-EGF, $7149 \pm 675$ pixels vs LacZ, $4230 \pm 331$ pixels, $P<0.001$; 4 weeks: HB-EGF, $6575 \pm 534$ pixels $v s$ LacZ, $4414 \pm 494$ pixels, $P<0.05)$ (Figure 8f).

Accordingly, histological examination revealed that individual cardiomyocytes at the border area were remarkably hypertrophic 2 and 4 weeks after Ad.HB-EGF injections (2 weeks: HB-EGF, $18.76 \pm$ $0.29 \mu \mathrm{m}$ vs LacZ, $16.53 \pm 0.34 \mu \mathrm{m} ; P<0.05 ; 4$ weeks: HB-EGF, $20.49 \pm 0.28 \mu \mathrm{m}$ vs LacZ, $18.23 \pm 0.40 \mu \mathrm{m}$, $P<0.05$ ) (Figures 9 and 10). Thus, overexpression of HB-EGF markedly induces cardiomyocyte hypertrophy and fibrosis without affecting cardiac function, suggesting that this molecule plays an important role in accelerating the remodeling process after MI.

\section{Characteristic Histological Findings in the MI Area}

To clarify the mechanisms responsible for the enhancement of post-MI remodeling in the hearts treated with the HB-EGF gene during the subacute and chronic stages of MI, we performed histological examinations of rabbit hearts at 2 and 4 weeks postMI. Increases in the number of cells in the MI area 2 and 4 weeks after MI were more prominent in rabbits receiving Ad.HB-EGF than in those receiving control Ad.LacZ (2 weeks: HB-EGF, 235 \pm 4 cells/ field vs LacZ, $145 \pm 4$ cells/field, $P<0.001 ; 4$ weeks: HB-EGF, $180 \pm 6$ cells/field vs LacZ, $97 \pm 3$ cells/ field, $P<0.001$ ) (Figure 11a-c). Likewise, the number of proliferating (Ki-67 positive) cells increased more in the Ad.HB-EGF-treated rabbits than in those treated with Ad.LacZ (2 weeks: HB-EGF, 27.6 \pm 1.0 cells/field vs LacZ, $7.6 \pm 0.3$ cells/field, $P<0.001 ; 4$ weeks: HB-EGF, 25.3 \pm 1.4 cells/field vs LacZ, $15.8 \pm 1.3$ cells/field, $P<0.001$ ) (Figure $11 \mathrm{~d}-\mathrm{f}$ ). Immunohistochemical studies demonstrated that these accumulated cells were primarily SMA-positive spindle myofibroblasts at both 2 and 4 weeks (2 weeks: HB-EGF, $62.7 \pm 0.8$ cells/field vs LacZ, $38.0 \pm 0.9$ cells/field, $P<0.001 ; 4$ weeks: HB-EGF, $48.6 \pm 1.7$ cells/field vs LacZ, $22.7 \pm 1.1$ cells/field, $P<0.001$ ) (Figure 11g-i), and RAM 11-positive macrophages at 2 weeks only (HB-EGF, 18.4 \pm 1.0 cells/field vs LacZ, $4.2 \pm 0.3$ cells/field, $P<0.001$ ) (Figure 11j-1). On the other hand, the finding that more CD31-positive vascular endothelial cells were observed in the border area than in the remote area in both groups suggests an angiogenic effect induced by certain endogenous factors following MI (Figure 11m-0).

Interestingly, these vascular endothelial cells were not further increased by Ad.HB-EGF injections, in contrast to the significant increases observed in total cells, proliferating cells, myofibroblasts and macrophages, suggesting that HB-EGF most likely lacks angiogenic potential. 

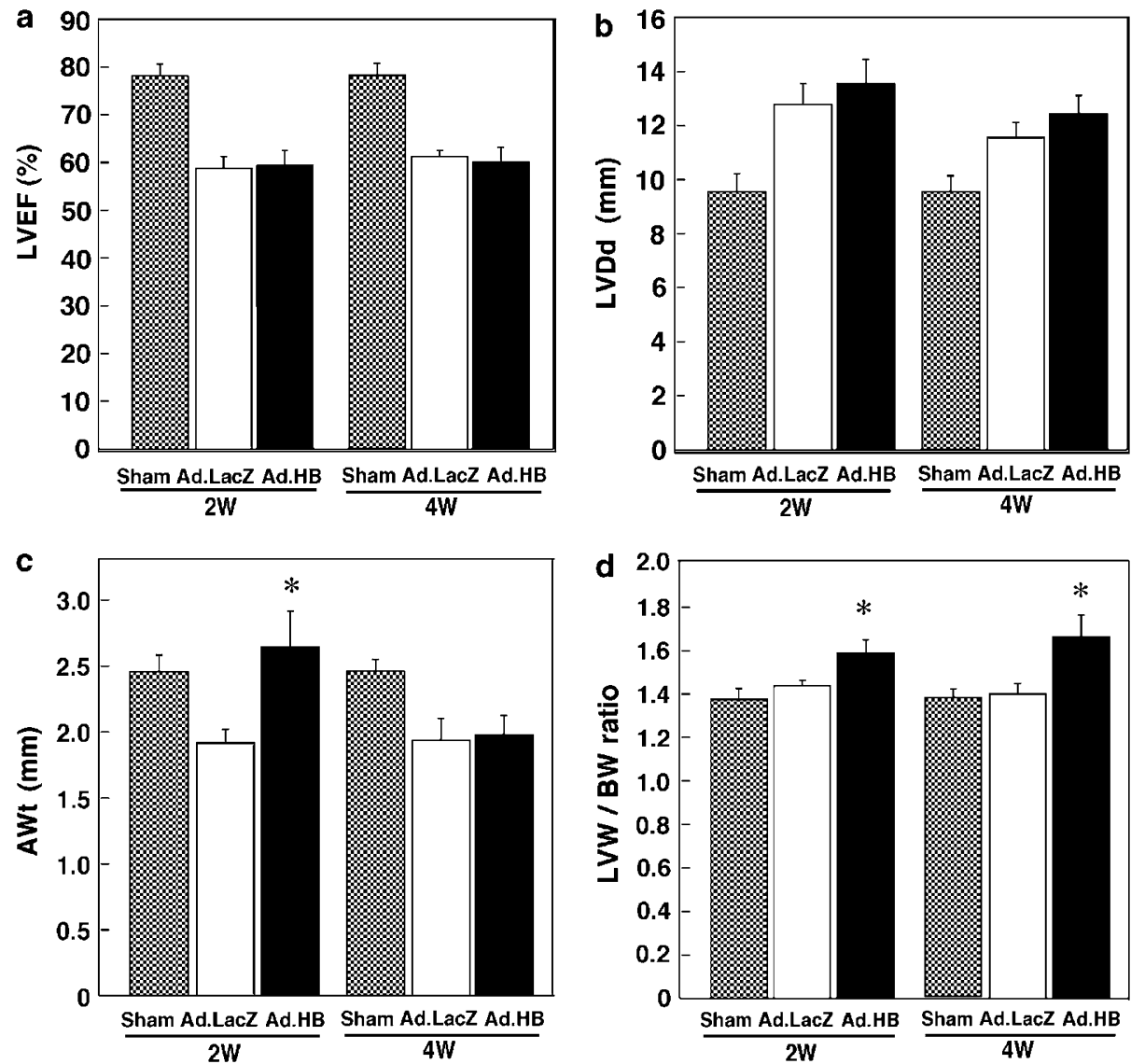

Figure 7 Echocardiographic measurements and left ventricular (LV) weight after adenoviral HB-EGF gene transduction in the postinfarct rabbit heart. Graphs showing the (a) LVEF, (b) LV size, (c) wall thickness, and (d) LV weight to body weight at 2 and 4 weeks after Ad.HBEGF gene transduction in MI rabbits. Cardiac function parameters were assessed by echocardiographic examination. LVEF: left ventricular ejection fraction; LVDd: left ventricular dimension at end-diastole; AWt: anterior wall thickness; LVW: LV weight; BW: indicated body weight. 'Sham' indicates sham-operated rabbits without MI or adenoviral transduction. ${ }^{*} P<0.05$.

\section{Apoptosis in the MI Area after Adenoviral HB-EGF Gene Transduction}

To estimate apoptosis in the MI area, TUNEL staining was performed. Unexpectedly, the number of TUNEL-positive cells was increased by Ad.HB-EGF injection at 2 weeks after MI (HB-EGF, $1.95 \pm 0.10 \%$ vs LacZ, $1.04 \pm 0.09 \%, P<0.001$ ) (Figure 12). Notably, most of the TUNEL-positive cells were costained with the anti-RAM11 antibody (Figure 13a and b), but not with anti-SMA antibody (Figure 13c), nor by markers for cardiomyocytes such as troponin I (Figure 13d). Moreover, TUNEL-positive signals were detected in the cytoplasm of some macrophages with intact nuclei. Thus, the TUNEL-positive cells may be not only apoptotic macrophages, but also viable macrophages that had phagocytosed other apoptotic cells (Figure 13b). Thus, in vivo HB-EGF gene transduction stimulated the activation of noncardiomyocytes, including macrophages, fibroblasts and myofibroblasts, but not endothelial cells, in and around the MI area, while at the same time inducing cardiac hypertrophy.

\section{Discussion}

This is the first study to explore directly the in vivo effects of overexpressed HB-EGF on heart remodeling after reperfused MI. In addition, our unique adenoviral gene transduction and overexpression approach allowed a preliminary assessment of the potential utility of HB-EGF in gene therapy. Overexpressed HB-EGF in the MI lesion did not result in a beneficial or therapeutic outcome, in contrast to results observed with HGF or IGF in rodent MI models, but rather exacerbated the remodeling process through the activation of specific types of noncardiomyocytes.

To identify the HB-EGF-related biological mechanism underlying heart failure, distinguishing the various phenotypic effects of HB-EGF from those of HGF and IGF may prove useful, because despite their differences all of these factors are essential cardiogenic growth factors as well as potent inducers of cardiac hypertrophy. ${ }^{27,29,41}$ The most important difference between HB-EGF and HGF/IGF, which accounts for the observed discrepancy, is 

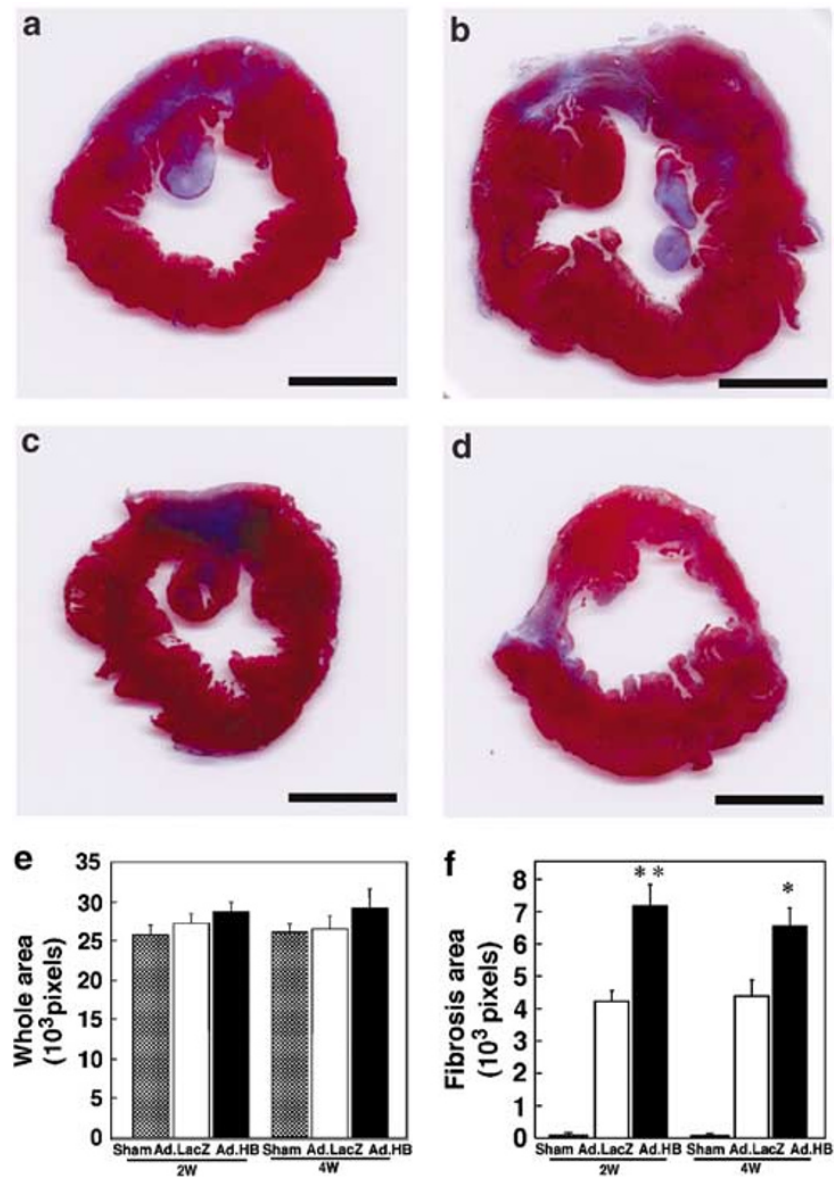

Figure 8 Macroscopic findings and histological analyses of transverse sections of hearts after adenoviral HB-EGF gene transduction. Fibrosis areas were stained blue with Masson's trichrome. (a) Ad.LacZ group (control) at 2 weeks after MI, (b) Ad.HB-EGF group at 2 weeks after MI, (c) Ad.LacZ group at 4 weeks after MI, (d) Ad.HB-EGF group at 4 weeks after MI. Graphs showing the whole areas (e) and fibrosis areas (f). The stained areas were morphometrically analyzed by counting pixels. Scale bar $=5 \mathrm{~mm},{ }^{*} P<0.05,{ }^{*} P<0.001$.

the lack of a direct cytoprotective effect of HB-EGF on cardiomyocytes, in contrast to the potent cytoprotective effect observed for HGF and IGF in injured hearts. ${ }^{27,29,41}$ This is a unique feature of HB-EGF, because most organogenic and/or organotrophic growth factors exert direct antiapoptotic effects, for example, HGF or IGF in mouse or rat cardiomyocytes ${ }^{26-29}$ and HB-EGF in the small intestine. ${ }^{42}$ In this regard, future studies to explore the differences among the molecules and signal transduction pathways involved in the activity of each growth factor would be biologically important.

Another important feature that differentiated HB-EGF from HGF and IGF was its observed lack of angiogenic activity. It should be noted that improvement of cardiac dysfunction after MI has been successfully achieved by gene therapy using angiogenic factors that do not directly act on
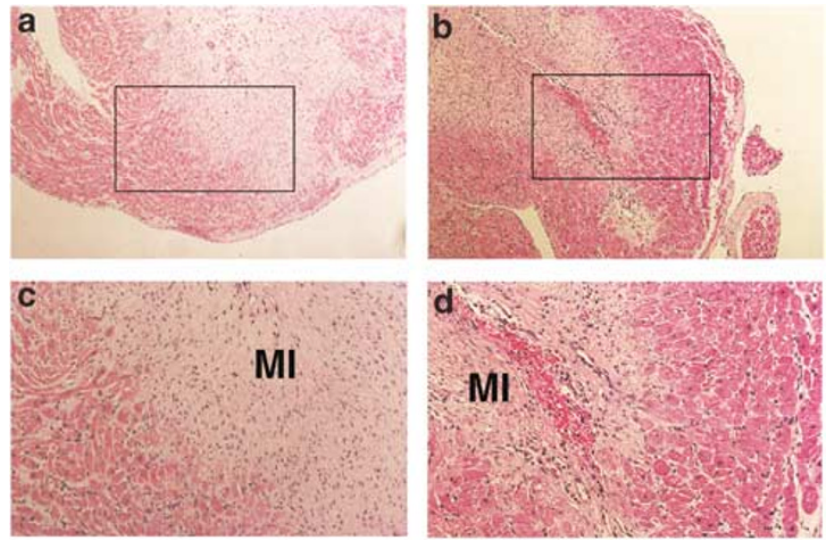

Figure 9 Histological findings at the MI border area after treatment. H-E-stained slides of LV 2 weeks post-MI. (a), (c) Ad.LacZ-treated rabbits and (b), (d) Ad.HB-EGF-treated rabbits. Squared-in areas in (a) and (b) (original magnification $\times 100$ ) were magnified in (c) and (d) (original magnification $\times 400$ ), respectively.

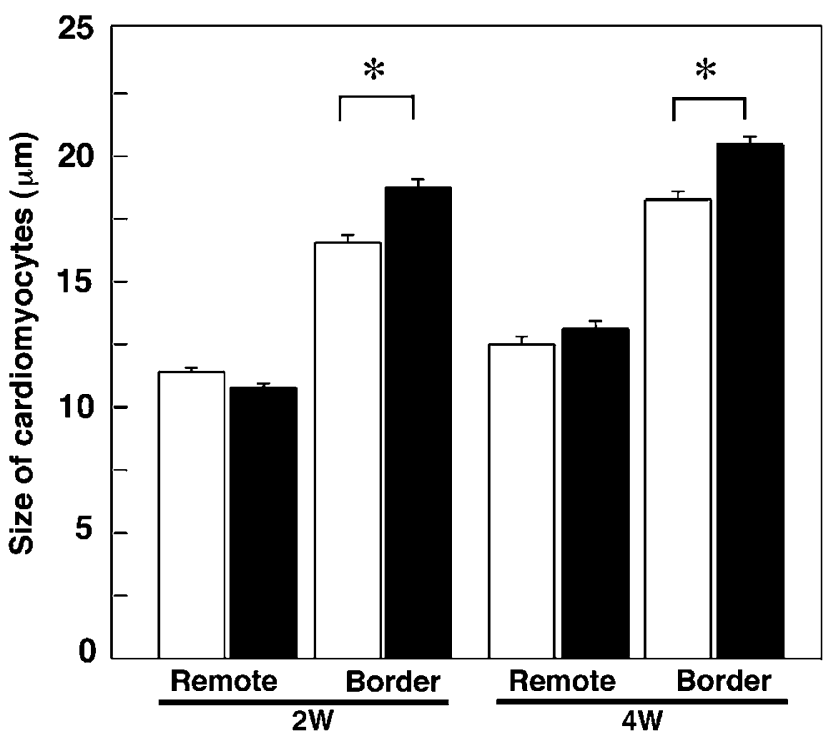

Figure 10 Size of cardiomyocytes at the remote and border areas of MI after treatment. The size of individual cardiomyocytes at the remote and border areas was morphometrically analyzed. ${ }^{*} P<0.001$.

cardiomyocytes. ${ }^{29,43}$ This fact suggests that angiogenesis plays a crucial role in postinfarction remodeling, and that the absence of an angiogenic effect of overexpressed HB-EGF may be largely responsible for its lack of therapeutic action.

In addition, HB-EGF was revealed to have a mitogenic effect on fibroblasts, in contrast to the potent antifibrotic effect of HGF following MI. ${ }^{27}$ Moreover, the characteristic finding after HB-EGF gene transduction was prominent accumulation of SMA-positive myofibroblasts and macrophages in the MI-affected areas. We previously reported that the infiltrating cells at the subacute stage post-MI 

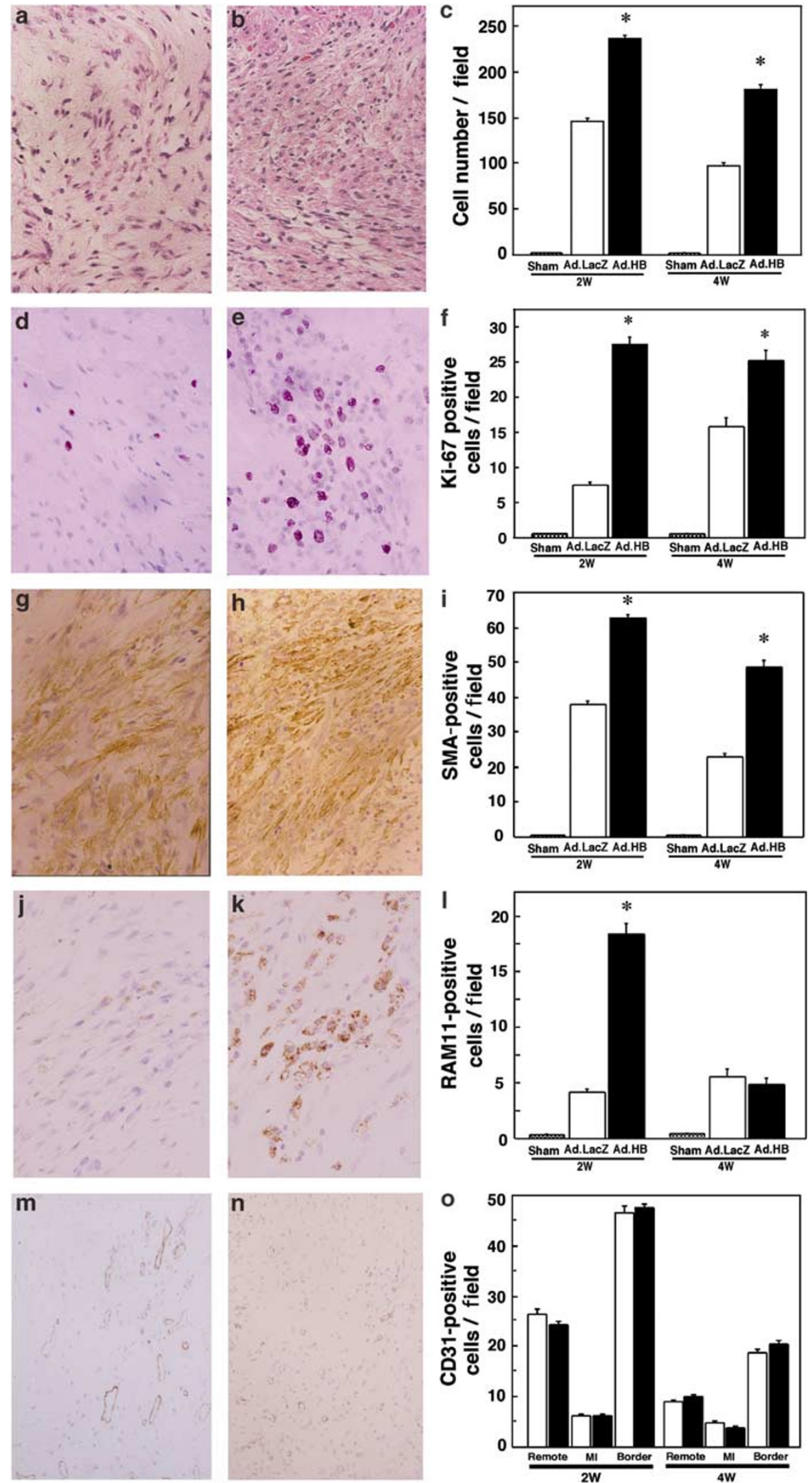

Figure 11 Histological and immunohistochemical findings in the MI area post-treatment. (a, b) H-E-stained-tissues, and immunohistochemically stained-tissues using (d, e) anti-Ki-67, (g, h) anti-SMA, (j, k) anti-RAM11, or (m, n) anti-CD31 antibodies 2 weeks after MI are shown (original magnification, $\times 400$ for $\mathbf{a}, \mathbf{b}, \mathbf{d}, \mathbf{e}, \mathbf{g}, \mathbf{h}, \mathbf{j}$ and $\mathbf{k}$, and $\times 100$ for $\mathbf{m}$ and $\mathbf{n}$ ). The number of positive cells in the field 2 and 4 weeks after MI were calculated and shown in the graphs (c, $\mathbf{f}, \mathbf{i}, \mathbf{l}$, and $\mathbf{o}) .{ }^{*} P<0.001$. 

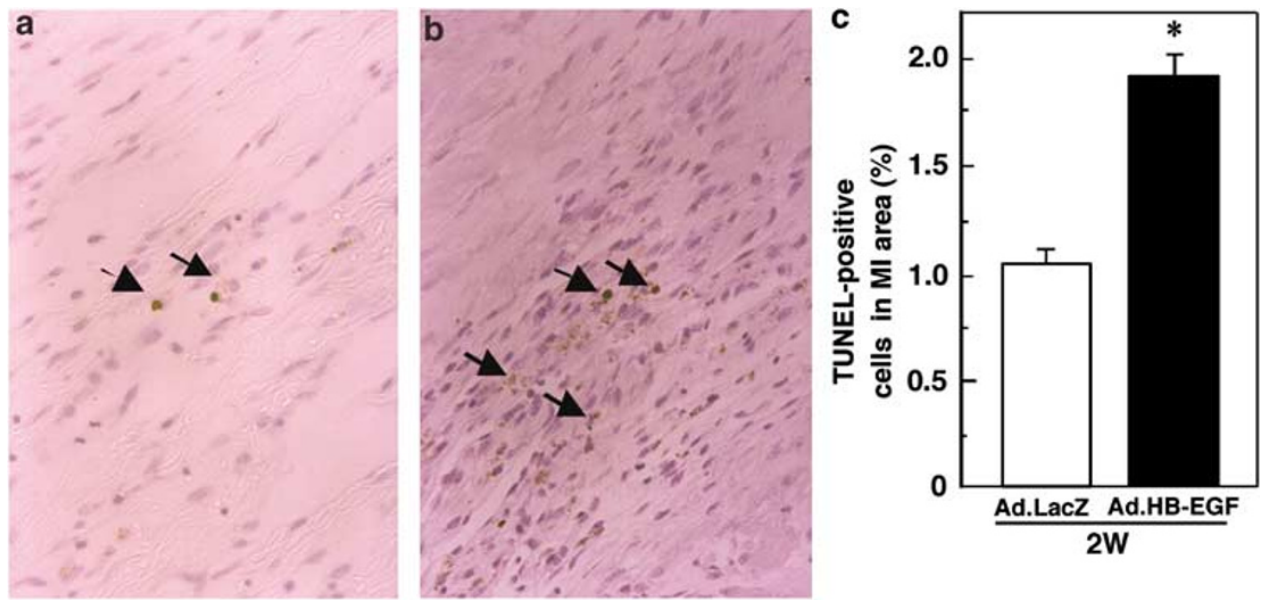

Figure 12 TUNEL staining in the MI area. TUNEL-positive cells (arrows) in the MI area 2 weeks after each treatment are shown. (a) and (b) indicate Ad.LacZ- and Ad.HB-EGF-treated rabbits, respectively. (c) The percentage of TUNEL-positive cells in the MI area was calculated by morphometric and quantitative analyses . ${ }^{*} P<0.001$.
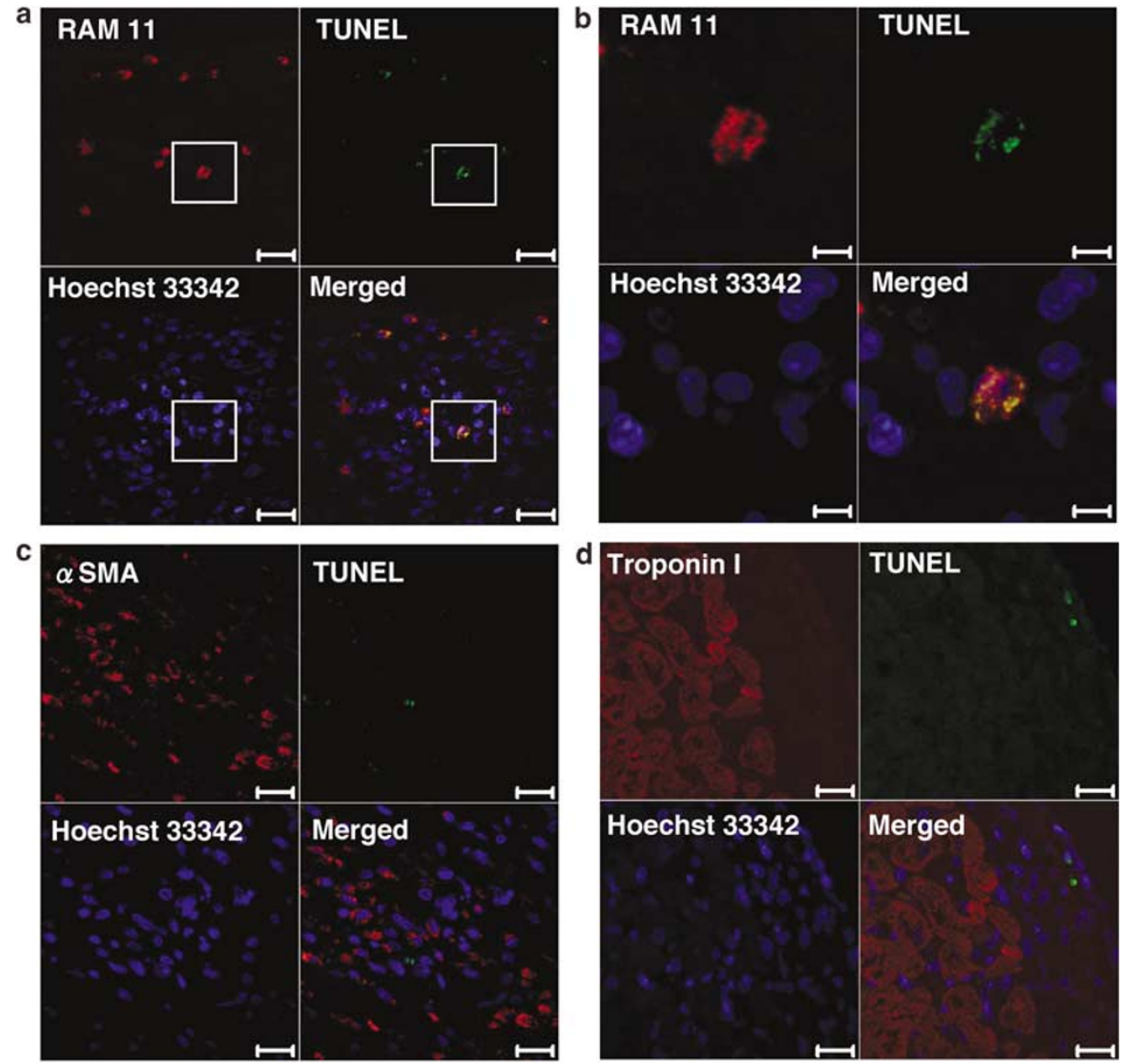

Figure 13 Immunofluorescent-TUNEL staining in the MI area. Laser confocal-microscopic analysis of slides that were triple-stained with TUNEL and (a, b) anti-RAM11, (c) anti-SMA and (d) anti-troponin I antibodies, and Hoechst 33342. Squared areas within (a) were magnified and shown in (b). Scale bars, $20 \mu \mathrm{m}(\mathbf{a}, \mathbf{c}, \mathbf{d})$ and $5 \mu \mathrm{m}(\mathbf{b})$.

were primarily macrophages, endothelial cells, and myofibroblasts, and that all of these cell types were entering apoptosis at an elevated rate. ${ }^{37}$ Thus, the previously unknown factor that activates fibroblasts, myofibroblasts, and macrophages during post infarction remodeling is now strongly suggested to be 
HB-EGF. Taken together with the lack of an agonistic death-inducible effect of HB-EGF directly on cardiomyocytes, pathologically upregulated HB-EGF may be responsible for activating these specific types of noncardiomyocytes, thus exacerbating post-MI remodeling.

The detailed molecular mechanisms by which HB-EGF activates these specific types of noncardiomyocytes remain to be elucidated. It was previously reported that HB-EGF stimulated the mitogenic and motogenic activities of smooth muscle cells,,${ }^{4,7}$ and also that it reduced the expression of SMA in fibroblasts. ${ }^{17} \mathrm{HB}-\mathrm{EGF}$ may play a regulatory role in the growth of fibroblasts and their transformation to myofibroblasts in the heart, as was suggested for the postinfarct kidney. ${ }^{17}$ However, the biological relationship between HB-EGF and macrophages has yet to be studied, so future work to explore these molecular mechanisms would be interesting and fruitful. In this study, the overexpressed HB-EGF may consist not only of soluble HB-EGF, a potent mitogen for diverse cell types, but also membranebound proHB-EGF, whose functions may be diverse depending on cell types. In this context, future biological studies comparing the physiological and pathological effects of proHB-EGF on the heart with those of soluble HB-EGF may be of interest, although a suitable experimental system should be carefully established.

Finally, the recent finding that shedding of proHBEGF resulted in cardiac hypertrophy suggests that upregulated HB-EGF might play a central role in hypertensive heart diseases. ${ }^{24,25}$ However, overexpression of HGF and IGF induced cardiac hypertrophy, but inconsistently exhibited potent therapeutic and beneficial effects on the injured heart, including that damaged by MI. ${ }^{27-29}$ Taken together with these facts, HB-EGF-induced cardiac hypertrophy may not be a sole or direct source of pathogenesis in MI, even though cardiac hypertrophy may, in fact, be involved in specific types of heart failure. In this context, the present results importantly imply that HB-EGFinduced exacerbation of remodeling may be a novel pathological mechanism for MI.

In conclusion, upregulated HB-EGF plays a pathological role in MI by activating specific types of noncardiomyocytes, leading to exacerbation of remodeling after MI. This novel fact may be useful for developing new therapeutics as well as for elucidating the mechanism of different types of heart failure, including MI.

\section{Acknowledgements}

This study was supported in part by Suzuken Memorial Foundation and a Grant-in-Aid for Scientific Research (C) from the Ministry of Education, Culture, Sports, Science and Technology of Japan. We thank Hatsue Oshika and Akiko Tsujimoto for their technical assistance.

\section{References}

1 Higashiyama S, Abraham JA, Miller J, et al. A heparinbinding growth factor secreted by macrophagelike cells that is related to EGF. Science 1991;251: 936-939.

2 Prenzel N, Zwick E, Daub H, et al. EGF receptor transactivation by G-protein-coupled receptors requires metalloproteinase cleavage of proHB-EGF. Nature 1999;402:884-888.

3 Ito N, Kawata S, Tamura S, et al. Heparin-binding EGFlike growth factor is a potent mitogen for rat hepatocytes. Biochem Biophys Res Commun 1994;198:25-31.

4 Higashiyama S, Abraham JA, Klagsbrun M. Heparinbinding EGF-like growth factor stimulation of smooth muscle cell migration: dependence on interactions with cell surface heparan sulfate. J Cell Biol 1993;122: 933-940.

5 Kiso S, Kawata S, Tamura S, et al. Liver regeneration in heparin-binding EGF-like growth factor transgenic mice after partial hepatectomy. Gastroenterology 2003; 124:701-707.

6 Iwamoto R, Mekada E. Heparin-binding EGF-like growth factor: a juxtacrine growth factor. Cytokine Growth Factor Rev 2000;11:335-344.

7 Miyagawa J, Higashiyama S, Kawata S, et al. Localization of heparin-binding EGF-like growth factor in the smooth muscle cells and macrophages of human atherosclerotic plaques. J Clin Invest 1995;95: 404-411.

8 Kalmes A, Daum G, Clowes AW. EGFR transactivation in the regulation of SMC function. Ann NY Acad Sci 2001;947:42-54; discussion 54-45.

9 Lemjabbar H, Basbaum C. Platelet-activating factor receptor and ADAM10 mediate responses to Staphylococcus aureus in epithelial cells. Nat Med 2002;8: 41-46.

$10 \mathrm{Fu} \mathrm{S}$, Bottoli I, Goller $\mathrm{M}$, et al. Heparin-binding epidermal growth factor-like growth factor, a v-Jun target gene, induces oncogenic transformation. Proc Natl Acad Sci USA 1999;96:5716-5721.

11 Marikovsky M, Breuing K, Liu PY, et al. Appearance of heparin-binding EGF-like growth factor in wound fluid as a response to injury. Proc Natl Acad Sci USA 1993;90:3889-3893.

12 Tokumaru S, Higashiyama S, Endo T, et al. Ectodomain shedding of epidermal growth factor receptor ligands is required for keratinocyte migration in cutaneous wound healing. J Cell Biol 2000;151:209-220.

13 Nguyen HT, Bride SH, Badawy AB, et al. Heparinbinding EGF-like growth factor is up-regulated in the obstructed kidney in a cell- and region-specific manner and acts to inhibit apoptosis. Am J Pathol 2000;156:889-898.

14 Sakai M, Zhang M, Homma T, et al. Production of heparin binding epidermal growth factor-like growth factor in the early phase of regeneration after acute renal injury. Isolation and localization of bioactive molecules. J Clin Invest 1997;99:2128-2138.

15 Xia G, Rachfal AW, Martin AE, et al. Upregulation of endogenous heparin-binding EGF-like growth factor (HB-EGF) expression after intestinal ischemia/reperfusion injury. J Invest Surg 2003;16:57-63.

16 Pillai SB, Hinman CE, Luquette $\mathrm{MH}$, et al. Heparinbinding epidermal growth factor-like growth factor protects rat intestine from ischemia/reperfusion injury. J Surg Res 1999;87:225-231. 
17 Kirkland G, Paizis K, Wu LL, et al. Heparin-binding EGF-like growth factor mRNA is upregulated in the peri-infarct region of the remnant kidney model: in vitro evidence suggests a regulatory role in myofibroblast transformation. J Am Soc Nephrol 1998;9: 1464-1473.

18 Iwamoto R, Yamazaki S, Asakura M, et al. Heparinbinding EGF-like growth factor and ErbB signaling is essential for heart function. Proc Natl Acad Sci USA 2003;100:3221-3226.

19 Jackson LF, Qiu TH, Sunnarborg SW, et al. Defective valvulogenesis in HB-EGF and TACE-null mice is associated with aberrant BMP signaling. EMBO J 2003; 22:2704-2716.

20 Nakamura Y, Handa K, Iwamoto R, et al. Immunohistochemical distribution of CD9, heparin binding epidermal growth factor-like growth factor, and integrin alpha3beta1 in normal human tissues. J Histochem Cytochem 2001;49:439-444.

21 Fujino T, Hasebe N, Fujita M, et al. Enhanced expression of heparin-binding EGF-like growth factor and its receptor in hypertrophied left ventricle of spontaneously hypertensive rats. Cardiovasc Res 1998; 38:365-374.

22 Iwabu A, Murakami T, Kusachi S, et al. Concomitant expression of heparin-binding epidermal growth factor-like growth factor mRNA and basic fibroblast growth factor mRNA in myocardial infarction in rats. Basic Res Cardiol 2002;97:214-222.

23 Tanaka N, Masamura K, Yoshida M, et al. A role of heparin-binding epidermal growth factor-like growth factor in cardiac remodeling after myocardial infarction. Biochem Biophys Res Commun 2002;297: 375-381.

24 Asakura M, Kitakaze M, Takashima S, et al. Cardiac hypertrophy is inhibited by antagonism of ADAM12 processing of HB-EGF: metalloproteinase inhibitors as a new therapy. Nat Med 2002;8:35-40.

25 Shah BH, Catt KJ. A central role of EGF receptor transactivation in angiotensin II-induced cardiac hypertrophy. Trends Pharmacol Sci 2003;24:239-244.

26 Taniyama Y, Morishita R, Aoki M, et al. Angiogenesis and antifibrotic action by hepatocyte growth factor in cardiomyopathy. Hypertension 2002;40:47-53.

27 Li Y, Takemura G, Kosai K, et al. Postinfarction treatment with an adenoviral vector expressing hepatocyte growth factor relieves chronic left ventricular remodeling and dysfunction in mice. Circulation 2003; 107:2499-2506.

28 Li Q, Li B, Wang X, et al. Overexpression of insulinlike growth factor-1 in mice protects from myocyte death after infarction, attenuating ventricular dilation, wall stress, and cardiac hypertrophy. J Clin Invest 1997;100:1991-1999.

29 Su EJ, Cioffi CL, Stefansson S, et al. Gene therapy vector-mediated expression of insulin-like growth factors protects cardiomyocytes from apoptosis and enhances neovascularization. Am J Physiol Heart Circ Physiol 2003;284:H1429-H1440.

30 Chen SH, Chen XH, Wang Y, et al. Combination gene therapy for liver metastasis of colon carcinoma in vivo. Proc Natl Acad Sci USA 1995;92:2577-2581.

31 Terazaki Y, Yano S, Yuge $\mathrm{K}$, et al. An optimal therapeutic expression level is crucial for suicide gene therapy for hepatic metastatic cancer in mice. Hepatology 2003;37:155-163.

32 Aoyama T, Takemura G, Maruyama R, et al. Molecular mechanisms of non-apoptosis by Fas stimulation alone versus apoptosis with an additional actinomycin D in cultured cardiomyocytes. Cardiovasc Res 2002;55: 787-798.

33 Ogasawara J, Watanabe-Fukunaga R, Adachi M, et al. Lethal effect of the anti-Fas antibody in mice. Nature 1993;364:806-809.

34 Takemura G, Kato S, Aoyama T, et al. Characterization of ultrastructure and its relation with DNA fragmentation in Fas-induced apoptosis of cultured cardiac myocytes. J Pathol 2001;193:546-556.

35 Toraason M, Wey $\mathrm{H}$, Woolery $\mathrm{M}$, et al. Arachidonic acid supplementation enhances hydrogen peroxide induced oxidative injury of neonatal rat cardiac myocytes. Cardiovasc Res 1995;29:624-628.

36 Villarreal FJ, Kim NN, Ungab GD, et al. Identification of functional angiotensin II receptors on rat cardiac fibroblasts. Circulation 1993;88:2849-2861.

37 Takemura G, Ohno M, Hayakawa Y, et al. Role of apoptosis in the disappearance of infiltrated, proliferated interstitial cells after myocardial infarction. Circ Res 1998;82:1130-1138.

38 Leor J, Quinones MJ, Patterson M, et al. Adenovirusmediated gene transfer into infarcted myocardium: feasibility, timing, and location of expression. J Mol Cell Cardiol 1996;28:2057-2067.

39 Barr E, Carroll J, Kalynych AM, et al. Efficient cathetermediated gene transfer into the heart using replicationdefective adenovirus. Gene Therapy 1994;1:51-58.

40 Chu D, Sullivan CC, Weitzman MD, et al. Direct comparison of efficiency and stability of gene transfer into the mammalian heart using adeno-associated virus versus adenovirus vectors. J Thorac Cardiovasc Surg 2003;126:671-679.

41 Huang CY, Hao LY, Buetow DE. Insulin-like growth factor-induced hypertrophy of cultured adult rat cardiomyocytes is L-type calcium-channel-dependent. Mol Cell Biochem 2002;231:51-59.

42 Michalsky MP, Kuhn A, Mehta V, et al. Heparinbinding EGF-like growth factor decreases apoptosis in intestinal epithelial cells in vitro. J Pediatr Surg 2001;36:1130-1135.

43 Siddiqui AJ, Blomberg P, Wardell E, et al. Combination of angiopoietin-1 and vascular endothelial growth factor gene therapy enhances arteriogenesis in the ischemic myocardium. Biochem Biophys Res Commun 2003;310:1002-1009. 